\title{
A New Role for the Parahippocampal Cortex in Representing Space
}

\author{
Sinéad L. Mullally and Eleanor A. Maguire \\ Wellcome Trust Centre for Neuroimaging, Institute of Neurology, University College London, London WC1N 3BG, United Kingdom
}

The debate surrounding the function of the human posterior parahippocampal cortex (PHC) is currently dominated by two competing theories. The spatial layout hypothesis proposes that PHC processes information about the shape of space embodied in layout-defining scene features. The contextual association hypothesis rejects this notion, proposing instead that PHC responds to highly contextualized, but not necessarily spatial, stimuli. Here we present a novel concept that suggests PHC is primarily concerned with any representation that depicts three-dimensional local space, be it scenes or even single objects. Specifically, we identified space-defining (SD) and spaceambiguous (SA) single objects, where SD objects consistently evoke a strong sense of the surrounding space while SA objects do not, in the absence of any background, spatial layout, or context. We found that participants could easily identify and distinguish between SD and SA objects. This distinction was subsequently affirmed at a neural level, where visualizing or viewing single SD objects compared with SA objects engaged PHC, despite these single SD objects offering no information about the shape or layout of the space. Moreover, this PHC response was robust and not accounted for by other factors, including contextual associations. Instead, it was linked to intrinsic object properties, specifically a combination of perceived object size and portability. By showing that PHC is responsive to the awareness of surrounding local space suggests its role in scene processing is basic and fundamental, such that it is not dependent on complex scene properties such as geometric structure, scene schema, or contextual associations.

\section{Introduction}

The posterior parahippocampal cortex (PHC) plays a role in a range of cognitive functions, including episodic memory, planning for the future, and spatial navigation (Spreng et al., 2009), but the precise function of this region is still debated. One prominent theory, the spatial layout hypothesis, proposes that $\mathrm{PHC}$ responds to the geometric structure of scenes embodied in layout-defining features such as walls and other immovable topographical elements (Epstein, 2008; Epstein and Ward, 2010). In this framework it is posited that scenes have special qualities over and above that of single objects, although these unique features remain ill defined (Epstein, 2008), and this ambiguity presents a problem for the spatial layout hypothesis.

Scenes are typically defined by the presence of multiple elements that exist in relationship to each other, resulting in a defined space (Biederman et al., 1982; Henderson and Hollingworth, 1999). The implication is that an awareness of the threedimensional (3D) space arises from the presence and arrangement of these elements. However, we suggest that a person's experience of $3 \mathrm{D}$ space can exist without the presence of multiple objects, large

\footnotetext{
Received Jan. 17, 2011; revised March 15, 2011; accepted April 4, 2011.

Author contributions: S.L.M. and E.A.M. designed research; S.L.M. performed research; S.L.M. and E.A.M. analyzed data; S.L.M. and E.A.M. wrote the paper.

This work was funded by the Wellcome Trust. Thanks to M. Symmonds and A. Nicolle for advice on data analysis and N. Weiskopf, E. Featherstone, A. Reid, J. Glensman, and D. Bradbury for technical assistance.

Correspondence should be addressed to Eleanor A. Maguire, Wellcome Trust Centre for Neuroimaging, Institute of Neurology, University College London, 12 Queen Square, London WC1N 3BG, United Kingdom. E-mail: e.maguire@fil.ion.ucl.ac.uk.

DOI:10.1523/JNEUROSCI.0267-11.2011

Copyright $\odot 2011$ the authors $\quad 0270-6474 / 11 / 317441-09 \$ 15.00 / 0$
}

scale structures, or a scene, and it is this basic sense of space that concerns the PHC. If this is true, then the spatial layout hypothesis would need to be extended to include the subjective experience of space even when evoked by single objects that are perceived or imagined in isolation, that is, devoid of a spatial layout or context.

To test this hypothesis, we identified a range of single objects that consistently evoked a strong sense of the surrounding space [space-defining (SD) objects] and other objects that had no such effect [space-ambiguous (SA) objects]. We assessed the validity of this novel SD/SA concept in a series of behavioral studies, followed by two functional MRI (fMRI) experiments. We hypothesized that the $\mathrm{PHC}$ would selectively respond to SD relative to SA objects. Such an observation would be contrary to previous studies that report minimal activation in PHC in response to discrete objects (Epstein and Kanwisher, 1998; Epstein et al., 1999) and would support the concept of the PHC as a basic spacespecific, as opposed to a scene-specific or place-specific, region.

A conflicting account of $\mathrm{PHC}$ function is the contextual association (CA) hypothesis, which suggests that the PHC is not specifically concerned with space, place, or scene processing but instead mediates the coactivation of associated representations (Bar and Aminoff, 2003; Bar, 2004). We considered whether engagement of the PHC could be accounted for by object contextualization. Thus, as well as investigating SD/SA objects, the stimuli were also characterized using Bar and Aminoff's (2003) CA criteria, and the neural response to this was assessed. While the CA hypothesis would predict increased activation of PHC for highly contextualized objects compared to objects only weakly associated with a specific context, we instead predicted greater activation of PHC for SD compared with SA objects, regardless 
of how strongly or weakly they were associated with a specific context.

\section{Materials and Methods}

There were three aspects to this study; first, a set of behavioral studies aimed at characterizing single objects along a range parameters. This was followed by an fMRI study and finally by another auxiliary fMRI study. The methods for each phase will now be described in turn.

\section{Behavioral experiments}

Participants. Fifty-one healthy, right-handed, native English speakers [29 females; mean age 25.62 years, standard deviation (StD) 3.53] participated in a series of five experiments $(\sim n=10$ per experiment). All had normal or corrected to normal vision and gave informed written consent in accordance with the local research ethics committee.

Stimuli and procedure. Descriptions of single objects were provided and participants had to imagine the objects based on these descriptions. This "imagination" method enabled us to more directly manipulate the cognitive process of interest, i.e., the internal representation of 3D space, by using stimuli that explicitly required participants to represent each stimulus as a 3D object within their mind's eye. However, to ensure that this imagination approach did not bias our findings, we also conducted parallel experiments where participants viewed pictures of single objects (see below, Auxiliary fMRI experiment).

We began with a pool of 399 everyday objects typically found within an indoor environment. Each object was accompanied by one or two descriptors (e.g., colors, patterns, material) to ensure its distinctiveness. During a training phase, participants were introduced to the concept of space-defining and space-ambiguous objects. It was suggested that certain objects, when imagined in isolation, evoke a strong sense of surrounding space (SD objects), while others do not (SA objects). Once it was clear the concept was understood and the importance of imagining the objects in isolation against a blank background was strongly emphasized, participants were presented with typed descriptions of each object (e.g., "a flowery cushioned sofa") and had to imagine the object in question as vividly as possible. Each object was imagined one at a time, without any surrounding objects or background contexts, and was then rated by the participant as either "space defining" or "space ambiguous." It was evident that participants were clearly and consistently able to distinguish between SD and SA objects. Objects rated as SD were assigned a score of 1 , while objects rated as SA were given a score of 0 . Those that could not be easily classified were assigned a score of 0.5 . Each object's cumulative score across participants was calculated and divided by the total number of participants (and multiplied by 100). Thus, if all participants rated an object as SD, it yielded a score of 100 , while an object rated as SA by all participants yielded a score of 0 [i.e., 100\% of participants rated the object as SA (0) and therefore not as SD (1)]. Using this SD/SA continuum, objects consistently categorized as either SD $(\geq 65 \%)$ or SA $(\leq 35 \%)$ were identified. After all objects had been rated in this way, participants were debriefed, i.e., participants were asked to describe the difference between an SD and an SA object and reflect on how the experience of imagining an SD object differed from the experience of imagining an SA object. Typical responses included: "SD items conjure up a sense of space whereas SA items float-they go anywhere." It is important to note that when participants used words such a "space" or "position" they were further probed to ensure that they were referring not to full or partial scenes, contexts, or places, but to space as a threedimensional but unspecific construct. Significantly, many spontaneously used the word "float" when discussing SA objects, emphasizing the detachment of these objects from an explicit sense of surrounding space. Next, the rating procedure was repeated, with participants now rating each object in terms of its size (i.e., was the specific object considered to be very small, small, medium, large, or very large). Size judgments were based solely on the participants' impression of each individual item's size and not relative to the other objects considered in the session. A number between 1 and 5 was then assigned to participants' size categorizations (i.e., "very small" $=1$, "very large" $=5$ ), and an average size rating (across participants) calculated for each object.
The primary purpose of phase one was to assemble a set of 250 objects for use in the subsequent fMRI experiment. Thus, 250 of the original 399 objects were selected-those that were most consistently rated as SD across subjects and those rated as consistently SA, while also ensuring, as far as possible, that the SD and SA object sets were similar in terms of their descriptors (i.e., colors, patterns, material) and size. This set of objects comprised 112 SD objects (e.g., "a large oak bed," "a dark corduroy couch," "an antique rocking horse"), 125 SA objects (e.g., "a large cardboard box," "a small white fan heater," "a wicker laundry basket"), and 13 objects that fell between the two categories (the latter included to facilitate linear parametric analyses).

Having established the SD/SA nature and the perceived size of these 250 objects, in the final set of behavioral studies, these objects were rated on a five-point scale across a range of other parameters, in particular to characterize them in terms of context. Specifically, participants rated how difficult it was to imagine an object in isolation $(1=$ very easy $\ldots .5=$ very difficult). Next they were asked to list any other objects which came to mind when the object was visualized (i.e., object-to-object associations). Items which consistently evoked thoughts of an associated object(s), were noted and a percentage score across participants was calculated. Finally, participants were asked to rate how often they would expect the object's position in a room to change in everyday life $(1=$ often $\ldots 5=$ rarely), yielding an object 'portability' score. They were also asked to name the place they most strongly associated the object with (i.e., object-to-context associations, using the method of Bar and Aminoff, 2003). As with the SD/SA ratings, each object's cumulative score across participants was calculated and divided by the total number of participants (and multiplied by 100). Thus, if all participants listed the same associated context, it yielded a score of 100 (and was considered to be highly contextualized), while if only half of the participants listed one particular context, that object yielded a score of 50 . To classify the objects into two groups (i.e., a highly contextualized group and a weakly contextualized group), we calculated the median score for the 250 objects (median $=56$ ). We thus considered objects with a score of 62 and above to be highly contextualized ( $n=114$; mean score, 82.63 ; StD, 10.91) and objects with a score of 50 and below to be weakly contextualized $(n=95$; mean score, 36.14; StD, 8.57). Following this, participants were asked whether it was possible to imagine the place they associated with an object without that object being present (i.e., expected presence, $1=$ no, not often $\ldots 5=$ always). This yielded a measure of how critical each object was to its associated context.

The mean scores on each object measure (and comparisons between SD and SA objects) are shown in Table 1). Overall, SD objects were significantly larger than SA objects $\left(t_{(235)}=20.04, p<0.001\right)$. Interestingly, there was some size overlap between SD and SA objects in the medium size category, suggesting that participants did not make SD/SA decisions based on size alone. Object portability, a measure of how often participants would expect an item's position to change in a room in everyday life, differed significantly between the SD and SA objects $\left(t_{(235)}=\right.$ $15.27, p<0.001)$, with SD objects being generally less portable than SA objects. Despite this overall difference, however, a considerable number of SA objects were also considered to have low portability (portability score $\geq 4 ; n=25$ ). This shows that participants did not make the SD/SA decision solely on the basis of object portability. Thus, the behavioral studies resulted in a set of objects that were characterized along a range of parameters: SD/SA, size, portability, object-to-context associations (places most associated with the object), expected presence (expected presence of an object in a particular context), difficulty imagining the object in isolation, and object-to-object associations (any additional objects that come to mind when thinking about this object).

\section{Primary fMRI experiment}

Participants. A new group of 21 healthy, right-handed, native English speakers, who had not taken part in any of the behavioral studies, participated in this fMRI experiment ( 8 females; mean age, 24.9 years; StD, 4.0). All participants had normal or corrected to normal vision and normal hearing and gave informed written consent in accordance with the local research ethics committee. Participants were unaware of the SD/SA distinction. Their naivety was critical to ensure an unbiased assessment 
Table 1. Mean ratings for object parameters

\begin{tabular}{|c|c|c|c|c|c|}
\hline & Description & Measure & SD objects & SA objects & \\
\hline \multicolumn{6}{|l|}{ Behavioral studies } \\
\hline Size & Imagined object size & (1) Very small ... (5) very large & $3.61(0.50)$ & $2.16(0.51)$ & $\dagger^{*}$ \\
\hline Portability & Imagined object movement & (1) Often moves ... (5) rarely moves & $4.56(0.61)$ & $2.95(0.95)$ & $\dagger^{*}$ \\
\hline Object-to-context associations & Place most strongly associated with the object & Consistency across participants (\%) & $65.12(22.45)$ & $55.27(22.87)$ & $\dagger^{*}$ \\
\hline Expected presence & Expected presence of an object in the associated context & (1) No, not often ... (5) always & $2.73(0.69)$ & $2.92(0.79)$ & NS \\
\hline Isolation & Difficulty imagining the object in isolation & (1) Very easy ... (5) very difficult & $1.97(0.42)$ & $1.86(0.52)$ & NS \\
\hline Object-to-object associations & Any additional object(s) that automatically come(s) to mind & Consistency across participants (\%) & $64.37(16.48)$ & $60.48(21.4)$ & NS \\
\hline \multicolumn{6}{|l|}{ Post-scan debriefing } \\
\hline Difficulty & Difficulty imagining the object & (1) Very difficult ... (5) very easy & $3.56(0.45)$ & $3.79(0.44)$ & $\ddagger^{*}$ \\
\hline Vividness & Vividness of the imagined object & (1) Not at all vivid...(5) very vivid & $3.42(0.55)$ & $3.66(0.56)$ & $\ddagger^{*}$ \\
\hline
\end{tabular}

Standard deviations in parentheses. ' Significantly more for SD than $S A$; ${ }^{*}$ significantly more for $S A$ than $S D\left({ }^{*} p<0.001\right)$.

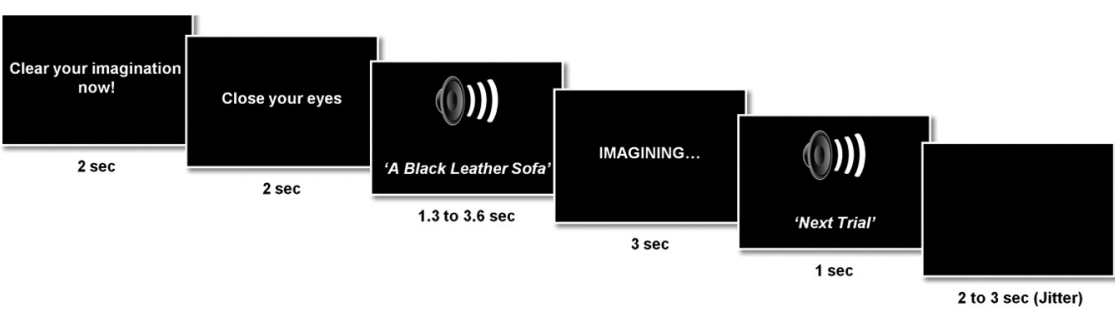

Figure 1. Task design. Timeline of an example SD trial. See Materials and Methods for full details. were not permitted to visualize any other items (other than what had been explicitly described) or to think of memories of similar items that had previously been encountered or other items that may be associated with the item being imagined. As discussed previously, each object and background item was accompanied by one or two descriptors (e.g., colors, patterns, material). These were balanced across SD and SA objects. A "next trial" auditory cue signaled the end of the visualization period. Following a jittered gap of $2,2.5$, or $3 \mathrm{~s}$, a new trial commenced.

of the neural basis of SD and SA objects. Thus, participants merely imagined (or viewed; see below, Auxiliary fMRI experiment) single objects during scanning, while unbeknownst to them, the objects had been consistently agreed by participants in the previous behavioral studies to be SD or SA.

Stimuli and procedure. Participants performed 350 trials in 5 scanning sessions of $\sim 10.5$ min each, with a $\sim 2$ min gap between each session during which the participant spoke with the experimenter (see below, Debriefing). Each session began with an explicit instruction to clear their imagination and to close their eyes (Fig. 1). The 250 objects from the behavioral studies described above (plus 50 background items-walls, floors) and 50 control jargon phrases (e.g., "a program question") (Summerfield et al., 2010) were presented auditorily to participants one at a time. Each item was presented only once during the experiment, and the order of conditions was randomized. Note that our main interest was in SD and SA objects; therefore, specific analyses relating to background items and control jargon phrases are not reported here (and did not involve activation of PHC).

Before scanning, participants received extensive training to ensure they fully understood the task requirements. In particular, participants were trained to clear their minds between each trial and to imagine each item devoid of any context. This latter point was emphasized throughout the training session, and participants did not proceed to the fMRI study until the experimenter was confident that they were imagining the practice items in isolation and devoid of any context. To ensure that this was not forgotten and to reinforce the importance of this requirement, participants were asked to rate how successful they felt they were at adhering to this instruction after each scan session $(1=$ very successful, $2=$ mostly successful, $3=$ fairly successful, $4=$ not that successful, $5=$ not at all successful). Participants' mean score on this rating was 2.56 (StD 0.3), showing they were successful at adhering to this instruction. In addition, participants were instructed to imagine each stimulus at a distance of $\sim 1 \mathrm{~m}$.

Visualization trials. After participants cleared their minds and closed their eyes, they were then required to visualize the object whose description they heard, in isolation and devoid of surrounding items or additional background contexts. The auditory item descriptions lasted $\sim 2 \mathrm{~s}$ (SD: mean, 2.11; StD, 0.30; range, 1.41-3.43; SA: mean, 2.10; StD, 0.34; range, 1.44-3.59; unsure: mean, 2.23; StD, 0.36; range, 1.75-2.84; backgrounds: mean, 2.10; $\mathrm{StD}, 0.26$; range, 1.46-2.78), and this was followed by a $3 \mathrm{~s}$ visualization period. It was emphasized that during this time they
Control trials. Control trials proceeded in the same way, but with participants hearing short jargon phrases (mean duration, 1.87; StD, 0.31; range, 1.26-2.40). Participants were instructed to attend to these phrases but not to visualize any related images or to recall any memories associated with the phrase content. Each jargon phrase was presented only once during the experiment.

Catch trials. To ensure that attention was maintained throughout the experiment, participants were informed they might be asked whether a certain word had been spoken during the preceding trial. This occurred on $10 \%$ of trials (including objects, backgrounds, and control trials). A catch trial was indicated by a different auditory cue (signaling to participants to open their eyes) in place of the next trial auditory cue. Participants were visually presented with one descriptor word (e.g., "black"). Their task was simply to decide whether this word was used in the previous object description (e.g., "a black leather sofa"; response = yes). Responses were recorded using an fMRI-compatible keypad. Participants had a maximum of $5 \mathrm{~s}$ to respond, after which they were instructed to close their eyes. The next trial audio signal was then played and the successive trial proceeded as normal. Catch trials never occurred on two successive trials and were excluded from any subsequent analysis.

Debriefing. Immediately following scanning, participants were debriefed and asked to rate each of the items that were presented during scanning with respect to difficulty imagining each item (from 1 to 5, low-high) and vividness (from 1 to 5, not at all vivid-very vivid) of the imagined image. The experimenter then discussed overall task performance with participants and asked them to rate (from 1 to 5 , low to high) aspects of the tasks in general, such as how difficult it had been to imagine the items in isolation (and against a blank background) and how frequently personal memories/other objects/backgrounds/structures came to mind when performing the task.

Scanning parameters and preprocessing. T2*-weighted echo planar images (EPI) with blood oxygen level-dependent (BOLD) contrast were acquired on a 1.5 tesla Siemens Sonata MRI scanner. Scanning parameters were selected to achieve whole brain coverage: 45 oblique axial slices angled at $30^{\circ}$ in the anterior-posterior axis; $2 \mathrm{~mm}$ thickness ( $1 \mathrm{~mm}$ gap); repetition time, $4.05 \mathrm{~s}$; slice time, $90 \mathrm{~ms}$; TE, $50 \mathrm{~ms}$; field of view, $192 \mathrm{~mm}$; $64 \times 64$ matrix; in-plane resolution; $3 \times 3 \mathrm{~mm}$. The first six "dummy" volumes from each session were discarded to allow for T1 equilibration effects. Field maps were acquired with a standard manufacturer's double echo gradient echo field map sequence (short $\mathrm{TE}=10 \mathrm{~ms}$; long $\mathrm{TE}=$ 
$14.76 \mathrm{~ms}$; whole brain coverage; voxel size, $3 \times 3 \times 3 \mathrm{~mm}$ ). A T1weighted structural scan was acquired for each participant with $1 \mathrm{~mm}$ isotropic resolution. Data were analyzed using the statistical parametric mapping software SPM5 (www.fil.ion.ucl.ac.uk/spm). Spatial preprocessing consisted of realignment and unwarping (using field maps), normalization to a standard EPI template in Montreal Neurological Institute (MNI) space with a resampled voxel size of $3 \times 3 \times 3 \mathrm{~mm}$, and smoothing using a Gaussian kernel with full width at half maximum of $8 \mathrm{~mm}$.

fMRI data analysis. After preprocessing, statistical analysis was performed using the general linear model. The experiment had three main "visualizing" conditions (space-defining, space-ambiguous, and background items) and one baseline control (jargon) condition. We modeled the time period from the start of the item description until the end of the gap (jitter) period (see Fig. 1 ) as a boxcar function of $\sim 9 \mathrm{~s}$ duration (exact durations were calculated on a per trial basis given the differences in stimulus durations and the jittered gap). We modeled this time period based on participants' comments in the debriefing session. Many participants reported that they continued to hold a clear image of the imagined object in their minds eye until the next trial cue, which initiated the start of the new trial, was heard. This was convolved with the canonical hemodynamic response function to create regressors of interest. Subject-specific movement parameters were included as regressors of no interest. Subject-specific parameter estimates pertaining to each regressor (betas) were calculated for each voxel. Second level random effects analyses were then run using one-sample $t$ tests on these parameter estimates (collapsed across sessions). We report the fMRI results at a voxel-level threshold of $p<0.001$, whole brain uncorrected (minimum cluster size of 5 voxels). We show all areas activated at this threshold. All activations are displayed on sections of the average structural MR image of all the participants. Reported voxels conform to MNI coordinate space. Right side of the brain is displayed on the right side.

\section{Auxiliary fMRI experiment}

While our main interest was in the imagination paradigm, we also conducted an additional fMRI study using pictures of real objects to verify that the SD/SA effect was not limited to the imagination protocol. In so doing we also took the opportunity to examine whether SD/SA effects were similar for indoor and outdoor objects. As in the imagination paradigm, a behavioral experiment was conducted before the fMRI study to select a set of stimuli categorized in terms of SD/SA.

Behavioral study. A group of 10 healthy, right-handed, native English speakers who had not been involved in any of the previous experiments participated ( 2 males; mean age, 25.3 years; StD, 4.3 years). All participants had normal or corrected to normal vision and normal hearing and gave informed written consent in accordance with the local research ethics committee. Using instructions identical to those described above (see above, Behavioral experiments), participants were first asked to rate 452 single objects in terms of SD and SA. Here, however, participants viewed two-dimensional pictures of the stimuli rather than imagining each object in their mind's eye. Objects were presented one at a time on a computer screen on a plain white background. The ratings were scored as in the imagination studies. The SD/SA distinction was readily made for pictures of both indoor and outdoor objects. Two hundred and eightyeight objects (consistently rated across subjects—144 SD objects: 96 indoor and 48 outdoor; 144 SA objects: 96 indoor and 48 outdoor) were selected to include in the fMRI component of this study.

In the second phase of this behavioral study, participants also rated each of the 177 stimuli used by Bar and Aminoff (2003) (obtained online from http://barlab.mgh.harvard.edu/ContextLocalizer.htm) as SD or SA. These ratings were scored as described previously.

fMRI study. A new group of 6 healthy right-handed native English speakers, who were unaware of the SD/SA distinction and had not taken part in the previous behavioral studies, participated in this fMRI study (5 male; mean age, 27.83 years; StD, 2.86 years). All participants had normal or corrected to normal vision and normal hearing and gave informed written consent in accordance with the local research ethics committee. Stimuli were visually presented in blocks with 6 stimuli per block (stimulus duration, $3000 \mathrm{~ms}$; interstimulus interval, $750 \mathrm{~ms}$ ), and there were 4 experimental conditions of interest (SD indoor objects, SA indoor ob- jects, SD outdoor objects, SA outdoor objects). In total, participants viewed 16 blocks of SD indoor objects and 16 blocks of SA indoor objects, 8 blocks of SD outdoor objects, and 8 blocks of SA outdoor objects. Ten blocks of scrambled images were also presented (scrambled using a $20 \times$ 20 grid in Adobe Photoshop C52). Blocks were presented in pseudorandom order with no two SD or SA blocks presented consecutively, and session order was counterbalanced across participants (there were two sessions). To ensure attention, participants had to perform a 1-back task throughout. There were four 1-back matches within each session, one match per condition (with the exception of the scrambled condition), which were subsequently excluded from the fMRI analysis. Scanning parameters, data preprocessing details, and statistical thresholds were identical to those described in the first fMRI study.

The experiment had two main conditions (SD and SA) each with two levels (indoor and outdoor) and one baseline (scrambled) condition. We modeled the time period from the start to the end of each block as a boxcar function of $\sim 22.5 \mathrm{~s}$ duration. This was convolved with the canonical hemodynamic response function to create regressors of interest. Subject-specific movement parameters were included as regressors of no interest. Subject-specific parameter estimates pertaining to each regressor (betas) were calculated for each voxel. Second level random effects analyses were then run using one-sample $t$ tests on these parameter estimates (collapsed across sessions). Our main interests were in the direct comparisons between SD and SA objects (SD $>$ SA), and between $\mathrm{SD}_{\text {indoor }}>\mathrm{SA}_{\text {indoor }}$ and $\mathrm{SD}_{\text {outdoor }}>\mathrm{SA}_{\text {outdoor }}$ objects.

\section{Results}

\section{Primary fMRI study}

The results of the behavioral experiments confirmed the validity of the SD/SA concept. Participants were able to categorize a large group of single objects using the criterion of awareness of the surrounding space, a characteristic that appears to encompass parameters such as object size and portability (see Materials and Methods and Table 1). Evoking a sense of the surrounding space is, however, a characteristic more traditionally attributed to scenes rather than single objects. We propose that SD objects are more similar to scenes than to SA objects when considered along this dimension. The aim of the next experiment was to assess, using fMRI, the brain areas associated with space-defining and space-ambiguous objects. We hypothesized that SD objects would activate scene-specialized brain areas such as PHC more than SA objects.

\section{Behavioral data}

Catch trials. Catch trials were performed during scanning. Participants performed with a high level of accuracy (mean, 97.7\%; StD, 2.7; range, 92-100), showing they maintained attention during the experiment. These trials were subsequently removed from the fMRI analysis.

Post-scan debriefing. Immediately following scanning, participants were asked to rate each of the items that were presented during scanning with respect to how difficult it was to imagine and the vividness of the imagined image. Mean per-object ratings for these two measures are shown in Table 1. These two ratings were used in the subsequent fMRI analysis, along with those obtained in the previous behavioral experiments (also shown on Table 1). Overall, SA objects were rated as significantly easier to imagine $\left(t_{(235)}=-4.06 ; p<0.001\right)$ and significantly more vivid $\left.t_{(235)}=-4 ; p<0.001\right)$ than SD objects.

In addition, participants were also asked to make some ratings not on a per-object basis but in terms of the overall experiment. These were not included in the fMRI analysis. Overall, participants found it relatively easy to imagine the objects in isolation against a blank background $(1=$ very easy $\ldots .5=$ very difficult; mean, 2.76; StD, 0.68), felt they were able to imagine the items 


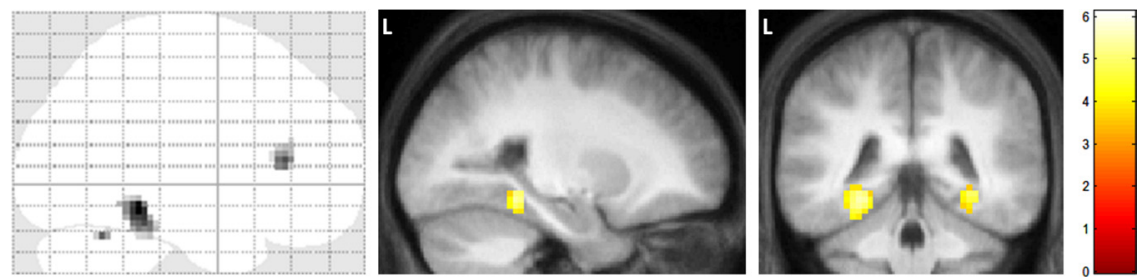

Figure 2. Brain areas engaged by imagining SD relative to SA objects. Brain areas more active for SD compared to SA objects are displayed (left) on a sagittal image from a "glass brain" that enables one to appreciate activations at all locations and levels in the brain simultaneously. Activations at the level of the peak left PHC voxel are shown on sagittal (middle) and coronal (right) images from the averaged structural MRI scan of the 21 subjects at a threshold of $p \leq 0.001$ (whole brain, uncorrected). The color bar indicates the Z-scores associated with each voxel. L, Left side of the brain.

without the addition of extra objects/background/structures $(1=$ kept to the description $\ldots 5$ = added lots of extra detail; mean, 2.19; StD, 0.60), avoided personal memories $(1=$ never . . . 5 = very frequently; mean, 2.29 ; StD , 1.01), and were able to concentrate well throughout the imagining sessions $(1=$ not focused at all . . . 5 = very focused; mean, 3.76; StD, 0.64).

\section{fMRI data}

Direct comparisons between SD and SA objects. Our main interest was in those brain areas that were selectively engaged when spacedefining items were imagined. We directly contrasted spacedefining objects with space-ambiguous objects $(\mathrm{SD}>\mathrm{SA})$ and observed activity in left PHC $(-27,-42,-12, Z=4.20$, cluster size $=61)$, right $\operatorname{PHC}(33,-39,-12, Z=4.54$, cluster size $=74)$, left middle frontal gyrus $(-51,33,12, Z=4.06$, cluster size $=$ 31 ), and right cerebellum $(6,-57,-24, Z=3.94$, cluster size $=$ 60 (Fig. 2). In a separate parametric analysis we defined an SD regressor using the cumulative space-defining ratings for each object, and activity linearly modulated by ratings that were increasingly space defining was identified (Wood et al., 2008). Similar results pertained when the data were modeled categorically (the data described here and in Fig. 2) or parametrically. In both models, the activity observed within PHC was in a region often referred to as the "parahippocampal place area" (PPA) (Epstein and Kanwisher, 1998). By contrast, the left lingual gyrus ( -18 , $-78,-6, Z=4.55$, cluster size $=22$ ), right superior temporal sulcus $(66,-30,6, Z=3.43$, cluster size $=12)$, right superior frontal gyrus $(6,33,48, Z=4.13$, cluster size $=10)$, left midcingulate cortex $(-12,-45,30, Z=3.25$, cluster size $=10)$, and left precuneus $(-3,-66,39, Z=3.29$, cluster size $=7)$ showed increased activity for SA objects compared with SD objects. Note that when only those objects that were rated as very easy/easy to imagine in isolation and so definitively devoid of filling in of background/spatial layout (94 SD objects and 99 SA objects) were used in the fMRI analysis, again robust bilateral activation of PHC was apparent (left PHC: $-30,-42,-9, Z=4.30$, cluster size $=41$; right PHC: $30,-36,-18, Z=3.70$, cluster size $=24$ ).

Contextual association hypothesis. It has been suggested that the PHC is specifically involved in the mediation of contextual associative processing (Bar, 2004). Could the SD-related PHC activity be accounted for by how contextualized the objects were, rather than in terms of how strongly they evoke an awareness of surrounding space? We addressed this issue in a number of ways. First, using the previously obtained measure of object-to-context associations (see Materials and Methods), we categorized the object stimuli as being either highly (114 objects) or weakly (95 objects) contextualized. By performing a categorical comparison between these two categories, we identified brain regions that responded to highly contextualized versus weakly contextualized objects. Greater activity was observed in left and right angular gyrus $(-45,-72$, $30, Z=3.42$, cluster size $=16 ; 42,-78$, $27, Z=3.69$, cluster size $=6 ; 39,-66,30$, $Z=5.54$, cluster size $=6$ ), left precuneus $(-12,-69,45, Z=3.71$, cluster size $=$ $10)$, right inferior frontal gyrus $(51,6,3$, $Z=3.86$, cluster size $=8)$, and left and right cerebellum $(-3,-72,-21, Z=$ 3.73, cluster size $=7 ;-18,-60,-27, Z=$ 3.60 , cluster size $=8 ; 33,-42,-39, Z=$ 3.49 , cluster size $=8 ; 9,-63,-18, Z=3.45$, cluster size $=5$ ) for highly contextualized objects; however, no activity changes in PHC were observed. To include all object stimuli in the analysis (and not simply the two extremes sampled in the "highly" and "weakly" contextualized object groups), we performed an additional parametric analysis using the cumulative "object-to-context associations" ratings for each object as a regressor of interest in the GLM. This revealed very similar regions of activity, and again no changes in PHC.

We thus failed to find evidence in support of the contextual association hypothesis. However, to ensure that we had thoroughly explored the data, we performed two additional analyses that sought to distinguish between activity modulated by linearly increasing levels of contextual associations from that attributable to the effect of SD (analysis 1), and activity linearly modulated by increasing SD independent of the effect of increasing contextual association (analysis 2). Thus, both the CA and SD scores were entered as regressors of interest in a GLM, and the second of these regressors was orthogonalized to the first (i.e., in analysis $1 \mathrm{CA}$ was orthogonalized to SD and in analysis 2 SD was orthogonalized to CA). This allowed the linear effect of increasing levels of a second orthogonalized regressor (i.e., CA in analysis 1 and SD in analysis 2) to be observed independent of the first regressor. No activity was observed in $\mathrm{PHC}$ for the independent component of the CA regressor (relative to SD) (Table 2), supporting our initial categorical and parametric CA analyses, which suggested that activity in the PHC was not evoked in response to highly as opposed to weakly contextualized objects. By contrast, the linear effect of increasing SD independent of CA (i.e., analysis 2) resulted in persistent activity in PHC bilaterally (Table 2) in response to this independent component of the SD regressor, suggesting that the effect in this region is driven by SD and not by contextual associations.

One possible explanation for the disparity between our findings and those of Bar and Aminoff (2003) is that their previous "highly contextualized" stimulus set may have contained a higher proportion of SD objects than the corresponding "weakly contextualized" set. We thus performed an additional behavioral study (see Material and Methods) where an independent group of participants made SD/SA ratings for each of the 177 stimuli used by Bar and Aminoff (2003). A significantly greater proportion of their highly contextualized stimulus set was rated as being $\mathrm{SD}$ in comparison to the proportion of stimuli considered to be SD in the weakly contextualized stimuli set: $t_{(9)}=4.565, p<0.01$ (Fig. 3).

The above analyses strengthen our original conclusion that the $\mathrm{PHC}$ responds robustly to the imagination of SD relative to SA objects, and this response is evident even when variance attributable to how contextualized the individual stimuli are is accounted for. Moreover, we found no evidence to suggest that contextual associations underpin the activity changes in 
Table 2. Brain areas modulated by increasing SD or contextual associations

\begin{tabular}{|c|c|c|}
\hline Region & \multicolumn{2}{|c|}{ Peak coordinates $(x, y, z)$} \\
\hline \multicolumn{3}{|c|}{ Linear effect of increasing CA (independent of SD) } \\
\hline Left cerebellum & $-18,-57,-30$ & 4.04 \\
\hline & $-6,-72,-21$ & 3.97 \\
\hline & $-12,-63,-24$ & 3.34 \\
\hline Left cerebellum & $-12,-81,-42$ & 3.60 \\
\hline Left insula & $-39,3,15$ & 3.94 \\
\hline \multicolumn{3}{|c|}{ Linear effect of increasing SD (independent of CA) } \\
\hline Right parahippocampal cortex & $33,-39,-12$ & 4.41 \\
\hline Left parahippocampal cortex & $-24,-42,-12$ & 4.15 \\
\hline Left middle frontal gyrus & $-48,30,12$ & 4.41 \\
\hline Right lateral occipital area & $51,-54,-12$ & 3.82 \\
\hline Left precentral sulcus & $-33,-3,51$ & 3.42 \\
\hline
\end{tabular}

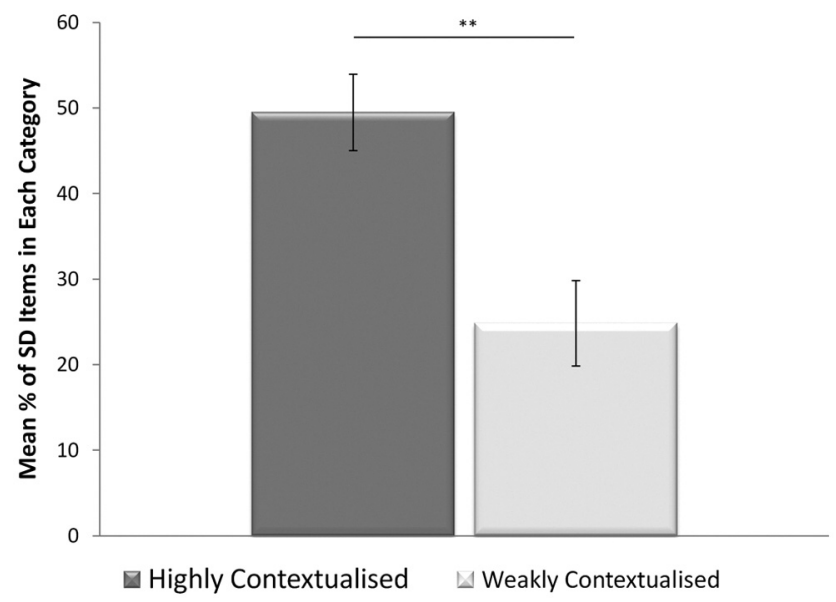

Figure 3. An analysis of Bar and Aminoff's (2003) stimuli in terms of SD/SA. Participants in one of our experiments rated a significantly larger proportion of objects as space defining in Bar and Aminoff's (2003) "highly contextualized" set of stimuli in comparison to their "weakly contextualized" set of objects. ${ }^{* *} p<0.01$.

the PHC and suggest instead that previous data supporting the CA hypothesis (e.g., Bar and Aminoff, 2003) was conflated with the effect of SD.

Factor analysis. Above, we examined the effects of SD that are independent of contextual associations but did not consider the influence of other potential variables on the SD effect. In total, we gathered data across nine separate dimensions for each object: $\mathrm{SD} / \mathrm{SA}$, object size, object portability, difficulty imagining the object, the vividness of the imagined object, difficulty imagining the object in isolation, object-to-object associations, object-tocontext associations, and expected presence of the object in an associated context (see Table 1). As some of these variables may potentially tap into the same underlying components, the scores on the nine parameters were submitted to principal components factor analysis. A varimax rotation was used to derive orthogonal factor scores with an eigenvalue of above 0.75. A total of four significant orthogonal factors were extracted that accounted for $83.86 \%$ of the variance in the data (Table 3). Uncorrelated factor score coefficients were estimated for each of the factors using the Anderson-Rubin method, and these were entered as regressors of interest in a GLM fMRI analysis.

SD ratings, object size, and object portability loaded strongly onto the first factor, and this factor accounted for $29.01 \%$ of the variance in the data (Table 3). The clustering of object size and portability with SD suggests these components are highly relevant to the SD concept. Interestingly, the brain regions modu-
Table 3. Results of the principal components factor analysis

\begin{tabular}{|c|c|c|c|c|c|}
\hline Variables & Factor 1 & Factor 2 & Factor 3 & Factor 4 & Variance (\%) \\
\hline SD ratings & 0.947 & -0.114 & 0.009 & 0.029 & 29.01 \\
\hline Size & 0.932 & -0.074 & 0.036 & 0.041 & \\
\hline Portability & 0.841 & 0.237 & -0.167 & -0.019 & \\
\hline Difficulty & -0.181 & 0.938 & -0.193 & 0.0817 & 23.32 \\
\hline Vividness & -0.163 & 0.941 & -0.159 & 0.052 & \\
\hline Isolation & 0.093 & -0.340 & 0.810 & 0.129 & 18.05 \\
\hline Object-to-object associations & 0.063 & -0.040 & 0.925 & 0.069 & \\
\hline Object-to-context associations & 0.208 & -0.135 & 0.132 & 0.824 & 13.48 \\
\hline \multirow[t]{2}{*}{ Expected presence } & -0.153 & 0.351 & 0.051 & 0.708 & \\
\hline & & & & & Total;83.86 \\
\hline
\end{tabular}

Boldface indicates each variable's highest factor loading.
A

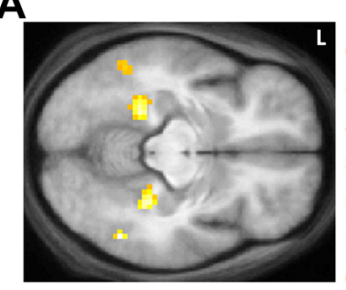

B

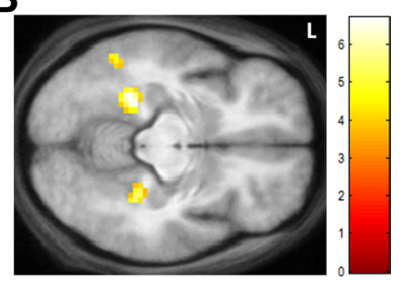

Figure 4. The brain areas engaged by the four factors extracted in the principal component analysis. $A$, Shown are the brain areas engaged by the linear effect of the "SD, size, and portability" factor extracted in first factor analysis and displayed at the peak voxel in left PHC. $\boldsymbol{B}$, Shown are the brain areas engaged by the linear effect of "size and portability" factor (i.e., excluding SD) extracted in second factor analysis and displayed at the peak voxel in left PHC. The activations are shown on the relevant axial sections from the averaged structural MRI scan of the 21 subjects at a threshold of $p \leq 0.001$ (whole brain, uncorrected). The color bar indicates the Z-scores associated with each voxel. L, Left side of the brain.

lated by this factor were remarkably similarly to those engaged by SD > SA (left PHC: $-24,-42,-12, Z=4.56,-30,-30,-21$, $Z=3.24$, cluster size $=64$; right PHC: $30,-36,-15, Z=4.70$, cluster size $=58$; left middle frontal gyrus: $-51,33,12, Z=4.90$, $-48,36,21, Z=3.50$, cluster size $=31$; left lateral occipital area: $-51,-51,-12, Z=3.38$, cluster size $=16$; right lateral occipital area: $51,-54,-12, Z=4.76$, cluster size $=9)($ Fig. $4 A)$. PHC was not modulated by any of the other three factors, including those that comprised the context parameters.

Given the strong relationship between SD, object size, and portability suggested by the factor analysis, we conducted a second factor analysis omitting the SD parameter and submitted the scores on the remaining eight parameters to a principal components factor analysis using the same technique as before. A total of four significant orthogonal factors were extracted that accounted for $83.65 \%$ of the variance in the data, suggesting that the addition of the SD parameter in the previous factor analysis had not resulted in greater explanatory power. Moreover, despite the exclusion of our SD parameter, object size and object portability both loaded onto the same discrete factor while ratings of object vividness and difficulty loaded together, as did difficulty imagining the object in isolation and object-to-object associations and object-to-context and expected presence. Finally, when the estimated uncorrelated factor score coefficients were entered as regressors of interest in a GLM, the brain regions modulated by the "object size and portability" factor were remarkably similarly to those modulated by both the SD > SA effect, and the "SD, object size, and object portability" factor from the first factor analysis (left PHC: $-24,-42,-12, Z=4.73$, cluster size $=67$; right PHC: $30,-36,-15, Z=4.34,30,-33,-24, Z=3.55$, cluster size $=$ 58; left middle frontal gyrus: $-51,33,15, Z=4.69,-45,39,24$, 


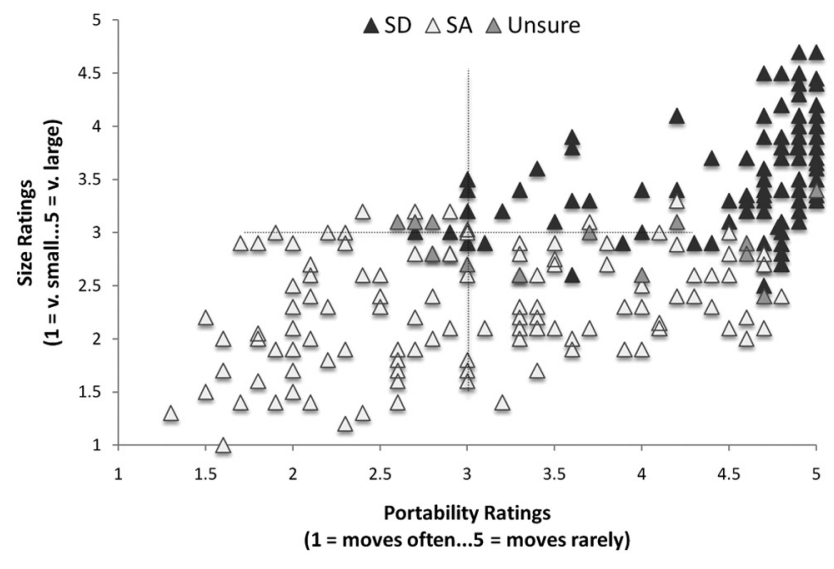

Figure 5. SD, object size, and object portability. Increasing "SD" correlated with both size ratings (very small ... very large) and object portability. Size and portability also correlated with one another. It is clear that the upper right quadrant contains many more SD objects, suggesting that a combination of larger size and less portability are intimately involved in the SD effect.
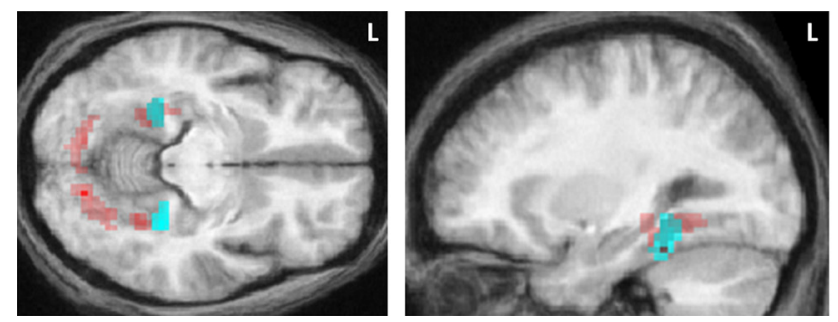

\section{Visual Paradigm}

Imagination Paradigm

Figure 6. Brain areas engaged by viewing pictures of SD relative to SA objects. Brain areas more active for SD compared with SA objects (collapsed across indoor and outdoor objects) in the "viewing" paradigm are shown in red on relevant axial and sagittal sections on the averaged structural MRI scan of the six subjects. The activations previously observed for the SD $>$ SA contrast in the "imagination paradigm (see Fig. 2) are displayed in blue, illustrating the overlap in the location of the activations between the two different presentation modes. All activations are shown at a threshold of $p<0.001$ (whole brain, uncorrected). L, Left side of the brain.

$Z=3.58$, cluster size $=31$; left lateral occipital area: $-51,-51$, $-12, Z=3.74$, cluster size $=15)($ Fig. $4 B)$. Thus, the combination of object size and portability appeared sufficient to produce robust and remarkably consistent activations in PHC.

Looking back to the behavioral studies (see Material and Methods and Table 1), it was apparent that SD objects were on average larger than SA objects, although there was a good deal of overlap in the medium size range for both object types, suggesting that size alone was not the cause of the SD effect. On average, SD objects were less portable, but again a considerable number of SA objects had low portability also, suggesting portability alone was not the basis of the SD effect. However, the results of the factor analyses suggest that it may be the combination of larger size and low portability that gives rise to the SD effect. The tightly coupled relationship between size and portability is confirmed in Figure 5, where increasing SD correlated with size ratings $(r=$ $0.87, p<0.001)$ and with ratings of object portability $(r=0.76$, $p<0.001$ ), while size and portability were correlated with one another $(r=0.70, p<0.001)$.

\section{Auxiliary fMRI experiment}

Finally, to verify that the SD effect in PHC was not merely specific to our imagination methodology, we conducted an additional fMRI study to validate the effect of SD within PHC using a more classical visual paradigm (see Materials and Methods). In these studies we also included indoor and outdoor objects to assess whether the SD effect pertained to both.

Examination of our original imagined objects fMRI data (Fig. 2) showed that the $S D>$ SA effect in PHC was remarkably robust even at the level of single subjects. Therefore, in this new fMRI study six participants viewed the object pictures. They had no knowledge of the SD/SA distinction but were occupied instead with performing a 1-back task [in line with similar paradigms in the object processing literature, e.g., Epstein and Kanwisher (1998) and Epstein and Ward (2010)]. Just as with the imaginastudy, the SD > SA effect for viewed objects was apparent in $\mathrm{PHC}$ at the same statistical thresholds as applied in the imagination study ( $p<0.001$ uncorrected) (left PHC: $-27,-39,-12$, $Z=4.72 ;-24,-42,-9, Z=4.28 ;-15,-39,-27, Z=3.90$, luster size $=103$; right PHC: $30,-48,-9, Z=4.40$, cluster 30) (Fig. 6, red activations). Moreover, the effects were remarkable overlap between PHC activations for the imagined objects (Fig. 6, blue activations) and the viewed objects (Fig. 6 , red activations), confirming the SD effect in PHC regardless of presentation mode. Finally, when the viewed objects were split into indoor and outdoor objects, the SD > SA effect in PHC was clearly evident for both contrasts, i.e., $\mathrm{SD}_{\text {indoor }}>\mathrm{SA}_{\text {indoor }}$ objects (left PHC: $-30,-33,-12, Z=4.48 ;-33$, $-42,-12, Z=3.79 ;-24,-33,-18, Z=$ 3.54 , cluster size $=29)$ and $\mathrm{SD}_{\text {outdoor }}>$ $\mathrm{SA}_{\text {outdoor }}$ objects (left PHC: $-24,-54$, $-3, Z=3.72 ;-24,-42,-12, Z=22$, cluster size $=22$ ).

\section{Discussion}

By introducing the concept of spacedefining and space-ambiguous single objects, we sought to divorce the basic awareness of local space from scene stimuli and their typical multicomponent structures. To do this, we used single objects categorized according to whether or not they elicited an awareness of surrounding space while ensuring that this did not include spatial layout or context. The results of the behavioral studies demonstrated that participants were capable of distinguishing between SD and SA objects. This distinction was subsequently affirmed at the neural level, where both the imagination and the viewing of single SD objects (indoor and outdoor) evoked activity within brain regions classically associated with the perception or imagination of full scenes (Epstein and Kanwisher, 1998; Aguirre and D'Esposito, 1999; O'Craven and Kanwisher, 2000; Maguire et al., 2001; Mendez and Cherrier, 2003; Hassabis et al., 2007; Park and Chun, 2009). This PHC response was robust and was not accounted for by a range of other potential explanatory factors such as contextual associations (Bar and Aminoff, 2003; Bar, 2004). Overall, this set of behavioral and neuroimaging experiments validate and characterize this novel concept of $\mathrm{SD} / \mathrm{SA}$ and suggest that the awareness of local $3 \mathrm{D}$ space may be a fundamental concern of the PHC.

Why is it necessary to respecify the role of the parahippocampal cortex in terms of the awareness of surrounding space, given that the $\mathrm{PHC}$ is already associated with processing of $3 \mathrm{D}$ geometric spatial layout (Epstein, 2008)? A central tenet of the spatial layout hypothesis purports that geometric spatial layout is derived from fixed elements, and this is absent in single objects 
(Epstein and Kanwisher, 1998; Epstein et al., 1999). Furthermore, while the PHC or "parahippocampal place area" as it is often called, is believed to respond to the geometry engendered by these elements, it remains unclear what it is about the geometric layout of a scene that the PHC actually responds to (for more on this problem, see Epstein, 2008). Here, we propose a more refined account of $\mathrm{PHC}$ function that suggests the $\mathrm{PHC}$ actually responds to the basic sense of three-dimensional space, necessarily present in scenes, but also inherently linked to some single object stimuli (i.e., space-defining objects). We propose that SD objects are unique in that they possess this spatial dimension while being independent of the typical multicomponent structure of scenes.

A significant aspect of our experiments was that participants were explicitly trained to imagine the individual objects in the absence of spatial layout or a larger scene context, and extensive efforts were made to ensure that participants continued to comply with this task instruction throughout the experiment. Feedback from the debriefing sessions, i.e., ratings from the behavioral experiments during and after the scanning sessions, confirmed that participants found it easy to imagine the objects in isolation (with no difference between SD and SA objects), felt themselves to be successful at doing so, and rarely included extra objects/ background/structures. Moreover, when we performed an fMRI analysis comparing only the "easily imagined in isolation SD objects" with the "easily imagined in isolation SA objects," as before, we found striking and robust bilateral parahippocampal cortex activations associated with SD compared with SA objects. Thus, when we refined our analysis to include only those objects easily visualized as singular isolated entities, we fully replicated our PHC finding. Thus, we can conclude with some confidence that the $\mathrm{PHC}$ responds robustly to single SD object stimuli and therefore to objects that evoke an awareness of surrounding space. Significantly, object stimuli in previous experiments have almost invariably been SA objects. No study has performed a truly unconfounded "scene $>$ SD object" comparison, and we would predict that any differential signal change between these stimulus categories would be a function of the amount of surrounding space evoked by individual stimuli. Thus, the concept of SD provides a more direct and concrete account of PHC function and removes the necessity to present whole scenes or places in stimuli that are difficult to decompose into their component parts. One could sum this up by suggesting that the parahippocampal place area might more accurately be referred to as the parahippocampal "space" area.

An obvious question that emerges from our findings is "what gives rise to the SD effect?" Our data offer some insight into this issue. The factor analyses showed that SD, object size, and object portability loaded onto the same factor, and the uncorrelated factor score coefficients were associated with robust PHC activation. Notably, when SD was omitted from the factor analysis, object size and portability again loaded together on the same factor, accounted for the same amount of experimental variance as the first factor analysis, and the uncorrelated factor score coefficients were associated with the same robust PHC activation. This strongly suggests that the PHC activations were driven by a combination of object size and portability. Examination of Figure 5 confirms that substantially more SD compared with SA objects were both larger and less portable. Thus, it seems likely that the $\mathrm{SD}$ effect, that is, where an object evokes a sense of surrounding space in the absence of spatial layout or context, is a consequence of object size and portability, and this is instantiated at the neural level in the PHC. SD objects anchor themselves in space, and the $\mathrm{PHC}$ response to SD objects is therefore biologically useful be- cause such objects are likely to have greater permanency and ultimately more utility in coding a space.

While our results strongly suggest the SD and PHC effect arises from a combination of object size and portability, our data also show that the PHC was not explicable in terms of contextual associations. As discussed previously, the contextual association hypothesis proposes that the $\mathrm{PHC}$ is engaged by the activation of previously acquired representations that link specific stimuli with particular (spatial or nonspatial) contexts (Bar and Aminoff, 2003; Bar et al., 2008). By categorizing our stimuli into those strongly associated with specific contexts (object-to-context associations), strongly and weakly contextualized objects could be directly compared. No evidence was found that the PHC was engaged by the processing of these contextualized stimuli or by the component of the CA regressor that was independent of SD. Similarly, other aspects of contextual processing ("expected presence in the associated context," "object-to-object associations," or "difficulty imaging the objects in isolation") also failed to activate the PHC. The factor analyses further confirmed that contextual parameters did not load onto the same factor as SD and, moreover, the factors onto which they loaded were not associated with PHC activation. Finally, when the SD regressor was examined with the effects of CA partialed out, the $\mathrm{PHC}$ effect remained robust.

Interestingly, several other studies have also failed to replicate the contextual association effect (Henderson et al., 2008; Epstein and Ward, 2010). Our data suggest a reason why Bar and Aminoff (2003) may have observed a PHC response to highly contextualized objects. We strongly suspected that there may have been an over-representation of SD objects in their highly contextualized category and an over-representation of SA objects in the weakly contextualized category within their stimulus set. We confirmed this bias (Fig. 3) in our behavioral study where participants rated the Bar and Aminoff (2003) stimuli in terms of our SD/SA categorization. The overrepresentation of SD objects in Bar and Aminoff's "highly contextualized" stimuli relative to their "weakly contextualized" stimuli set suggests their previous high/weak contextualization manipulations were confounded by SD.

In conclusion, our findings add to the growing body of literature about the function of the parahippocampal cortex. Showing that some single objects define the space around them, and that the $\mathrm{PHC}$ is responsive to this awareness of surrounding local space, suggests that the role for $\mathrm{PHC}$ in scene processing is basic and fundamental, such that it is not dependent on more complex scene properties such as geometric structure (Epstein, 2008), scene schema (Mandler and Parker, 1976; Biederman et al., 1982; Bar and Ullman, 1996) or contextual associations (Bar and Aminoff, 2003; Bar, 2004; Bar et al., 2008). This implies that the spatial layout hypothesis (Epstein, 2008; Epstein and Ward, 2010) might be refined by accounting for our findings and, in doing so, this could help to overcome its current difficulties in defining exactly what it is about scenes that evokes a PHC response. Future studies are needed to examine whether and under what circumstances objects can become space defining and how the SD effect relates to other processes such as those concerning the navigational relevance of objects/landmarks (Janzen and van Turennout, 2004). How the SD-related PHC response interacts with other brain areas like the retrosplenial cortex (Vann et al., 2009) and regions concerned with scene construction such as the hippocampus (Hassabis and Maguire, 2007, 2009) will also need to be clarified. In the meantime, our findings underline the need for care in the design of future studies involving objects and scenes to ensure that the powerful SD effect in PHC is not misinterpreted. 


\section{References}

Aguirre GK, D'Esposito M (1999) Topographical disorientation: a synthesis and taxonomy. Brain 122:1613-1628.

Bar M (2004) Visual objects in context. Nat Rev Neurosci 5:617-629.

Bar M, Aminoff E (2003) Cortical analysis of visual context. Neuron 38:347-358.

Bar M, Ullman S (1996) Spatial context in recognition. Perception 25:343-352.

Bar M, Aminoff E, Schacter DL (2008) Scenes unseen: the parahippocampal cortex intrinsically subserves contextual associations, not scenes or places per se. J Neurosci 28:8539-8544.

Biederman I, Mezzanotte RJ, Rabinowitz JC (1982) Scene perception: detecting and judging objects undergoing relational violations. Cogn Psychol 14:143-177.

Epstein RA (2008) Parahippocampal and retrosplenial contributions to human spatial navigation. Trends Cogn Sci 12:388-396.

Epstein RA, Ward EJ (2010) How reliable are visual context effects in the parahippocampal place area? Cereb Cortex 20:294-303.

Epstein R, Kanwisher N (1998) A cortical representation of the local visual environment. Nature 392:598-601.

Epstein R, Harris A, Stanley D, Kanwisher N (1999) The parahippocampal place area: recognition, navigation, or encoding? Neuron 23:115-125.

Hassabis D, Maguire EA (2007) Deconstructing episodic memory with construction. Trends Cogn Sci 11:299-306.

Hassabis D, Maguire EA (2009) The construction system of the brain. Philos Trans R Soc Lond B Biol Sci 364:1263-1271.

Hassabis D, Kumaran D, Maguire EA (2007) Using imagination to understand the neural basis of episodic memory. J Neurosci 27:14365-14374.

Henderson JM, Hollingworth A (1999) High-level scene perception. Annu Rev Psychol 50:243-271.
Henderson JM, Larson CL, Zhu DC (2008) Full scenes produce more activation than close-up scenes and scene-diagnostic objects in parahippocampal and retrosplenial cortex: an fMRI study. Brain Cogn 66:40-49.

Janzen G, van Turennout M (2004) Selective neural representation of objects relevant for navigation. Nat Neurosci 7:673-677.

Maguire EA, Frith CD, Cipolotti L (2001) Distinct neural systems for the encoding and recognition of topography and faces. Neuroimage 13:743-750.

Mandler JM, Parker RE (1976) Memory for descriptive and spatial information in complex pictures. J Exp Psychol Hum Learn 2:38-48.

Mendez MF, Cherrier MM (2003) Agnosia for scenes in topographagnosia. Neuropsychologia 41:1387-1395.

O'Craven KM, Kanwisher N (2000) Mental imagery of faces and places activates corresponding stiimulus-specific brain regions. J Cogn Neurosci 12:1013-1023.

Park S, Chun MM (2009) Different roles of the parahippocampal place area (PPA) and retrosplenial cortex (RSC) in panoramic scene perception. Neuroimage 47:1747-1756.

Spreng RN, Mar RA, Kim AS (2009) The common neural basis of autobiographical memory, prospection, navigation, theory of mind, and the default mode: a quantitative meta-analysis. J Cogn Neurosci 21:489-510.

Summerfield JJ, Hassabis D, Maguire EA (2010) Differential engagement of brain regions within a 'core' network during scene construction. Neuropsychologia 48:1501-1509.

Vann SD, Aggleton JP, Maguire EA (2009) What does the retrosplenial cortex do? Nat Rev Neurosci 10:792-802.

Wood G, Nuerk HC, Sturm D, Willmes K (2008) Using parametric regressors to disentangle properties of multifeature processes. Behav Brain Funct 4:38. 